\title{
A Spatial Fluid-based Framework to Analyze Large-Scale Wireless Sensor Networks*
}

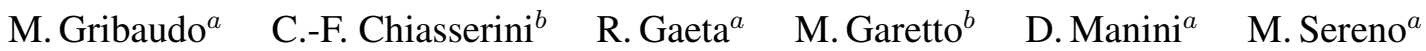 \\ ${ }^{a}$ Dip. di Informatica, Università di Torino $\quad{ }^{b}$ Dip. di Elettronica, Politecnico di Torino
}

\begin{abstract}
The behavior of large-scale wireless sensor networks has been shown to be surprisingly complex and difficult to analyze, both by empirical experiment and simulation. In this paper we develop a new analytical model of the behavior of wireless sensor networks, based on a fluid approach, i.e., we represent the sensor network by a continuous fluid entity distributed on the network area. The model accounts for node energy consumption, channel contention, as well as traffic routing; thus, it is well suited for describing the properties of sensor networks and understanding their complex behavior.
\end{abstract}

\section{Introduction}

Wireless sensor networks consist of hundreds to thousands of sensor nodes with limited computational and energy resources. Sensors are densely deployed over an area of interest, where they gather and disseminate local data using multihop communications, i.e., using other nodes as relays. A typical network configuration includes a large collection of stationary sensors operating in an unattended mode, which need to send their data to a node collecting the network information, the so-called sink node.

Traditionally, network designers have employed either computer simulations or analytical frameworks to predict and analyze systems behavior. Modelling large sensor networks, however, raises several challenges due to scalability problems and high computational costs. With regard to simulations, several software tools have been extended or developed on purpose to deal with large wireless networks, see $[1,2,3]$ just to name a few. As for analytical modelling, to the best of our knowledge, the only work dealing with large sensor networks is presented in [4], which employs percolation techniques.

In this paper, we present an novel analytical model for wireless sensor networks, based on a fluid approach. We

*This work has been partially supported by the Italian Ministry of Education, University and Research within the projects Perf (FIRB) and Pattern (PRIN). name our approach as spatial, fluid-based approach, because we represents the sensor nodes as a fluid entity. Sensor location is smoothed out in continuous space by introducing the concept of local sensor density, i.e., the number of sensors per area unit at a given point. We focus on a network whose nodes are static and need to send the result of their sensing activity to a sink node. Sensors may send packets to the sink in a multihop fashion. Although we introduce some simplifying assumptions that are necessary to maintain the problem tractable, our model accounts for: node energy consumption, node contention over the radio channel, and traffic routing. With respect to previous work, we provide three fundamental contributions:

(i) thanks to our fluid approach, very large networks can be studied while maintaining the model complexity extremely low;

(ii) the behavior of the network can be studied as a function of the bi-dimensional spatial distribution of the nodes, possibly under non-homogeneous node deployment;

(iii) the model provides a very flexible and powerful tool, which can account for various routing strategies, sensor behaviors, and network control schemes, such as congestion control mechanisms.

\section{Related Work}

In the recent literature, several analytical studies for sensor networks have been proposed. Theoretical studies of strategies for data dissemination and data collection are presented in [5, 6]. In [7, 8], forwarding techniques based on geographical location of the sensors are considered, and an analytical evaluation is presented. The problem of correlated data gathering has been also largely addressed. As an example, the work in [9] considers a network with a tree structure and tries to minimize the transmission cost of transferring data from sensors to a sink node by applying source coding techniques. An analytical approach to coverage and connectivity is employed in $[10,11,12]$. The 
work in [10] considers sensor grids, where sensors are unreliable and fail with a certain probability leading to random grid networks. In [11] coverage and object detectability of large sensor networks are investigated; while, in [12] percolation theory is used to study the impact of interference on the connectivity of large-scale ad hoc networks. A study on the transport capacity of sensor networks can be found in [13], where the authors consider simultaneous, multiple random processes that are detected by the nodes and need to be reconstructed at a sink node.

The closest works to ours are in [14, 4]. The work in [14] describes a Markovian model exploring the performance of a wireless network whose sensors switch between an active and a sleep mode, in order to save energy. The model is used to explore the network performance in terms of data latency, network throughput and energy consumption. The model accounts for traffic routing and channel contention, but its complexity does not allow to represent very large networks. A similar network scenario is studied in [4], under the assumption that only a few sensors are active at a time, and nodes broadcast any data they sense or receive to all their neighbors. The authors derive bounds on data latency by using percolation theory.

\section{Network Scenario and Assumptions}

Consider a network composed of $N$ sensor nodes randomly placed over an area in the plane, according to an independent and identical distribution, and their positions do not change over time. The sink node collecting all information gathered by the sensors can be placed at an arbitrary position within the network area.

Our assumptions on the sensor nodes are as follows.

(i) All nodes have a common, maximum radio range equal to $d$. Thus, any pair of nodes, say $(i, j)$, can communicate if they are within distance $d$ from each other, i.e., $\operatorname{dist}(i, j) \leq d$. Upon establishment of a link between $i$ and $j, i$ properly sets its transmission power so that its transmission can be successfully received by $j$ (no on-line power control is available, though ${ }^{1}$ ).

(ii) For the sake of simplicity, the node interference radius is set to $d$, i.e., the signal transmitted by the generic node $i$ will possibly 'disturb' all nodes within distance $d$ from $i$. Also, we assume that all nodes have a common sensing range that is equal to their maximum radio range.

(iii) The communication channel is assumed to be errorfree, although a channel error process could be easily included in the model.

${ }^{1}$ This is a fair assumption since typically sensor nodes are simple, lowcost devices (iv) The network topologies that we consider are always connected, i.e., there exists at least one path connecting each sensor to the sink. This assumption could be easily removed to investigate connectivity properties of the network, however we do not address this issue in this work.

(v) Each sensor generates data packets of constant size, which can be stored in a buffer while waiting to be transmitted. The buffer of each sensor is modelled as a FIFO queue. Also, by considering that the buffer is properly dimensioned so that the loss probability due to buffer overflow is negligible, we can assume infinite buffer capacity.

(vi) A sensor consumes a power equal to $P_{i}$ while being idle; the energy expenditure due to a one-hop communication is modelled as follows. Given the transmitterreceiver pair $(i, j)$, the energy consumed by $i$ to transmit a packet to $j$ is equal to $E^{(t x)}(i, j)$; the energy consumed by $j$ to receive a packet is equal to $E^{(r x)}$. Note that a sensor cannot simultaneously transmit and receive, and the energy expenditures in transmit/receive mode are additional w.r.t. the energy spent by a sensor while being idle.

(vii) To deliver their data, sensors may use multihop communications. We assume that each sensor independently sends data along the minimum-energy shortest path to the sink. To compute the shortest path, we apply the standard Dijkstra algorithm, assigning an energy cost $\epsilon$ to each link connecting two nodes that are within distance $d$ from each other. The cost $\epsilon(i, j)$ represents the total energy required to transfer a packet from node $i$ to node $j$ (one-hop energy cost), and is expressed by the sum of the cost at the transmitter and the cost at the receiver, $\epsilon(i, j)=E^{(t x)}(i, j)+E^{(r x)}$.

(viii) We represent channel contention by modelling a successful transmission over one hop similarly to the Protocol Model defined in [16]. Assume that node $i$ wants to transmit to $j$ (with $\operatorname{dist}(i, j) \leq d$ ); $i$ 's transmission to $j$ will be successful if no transmission from any sensor interfering with $j$ is taking place at the same time. Thus, a transmission from node $i$ to node $j$ is successful if, for every other node $k$, simultaneously transmitting, we have $\operatorname{dist}(k, j)>(1+\Delta) d$. The quantity $\Delta>0$ accounts for situations where a guard zone is specified by the protocol to prevent a nearby node from accessing the channel while being busy [16]. Note that, according to this model, whenever the generic node $i$ seizes the channel and no other sensor is transmitting within the radio range of $i$ 's intended receiver, the packet transmission is successful with probability 1 , i.e., it is not affected by interference at the physical 
layer, as is when CSMA-based access protocols are used. Upon a transmission failure, a node will attempt at retransmitting its packet until it is successfully delivered to the receiver; while waiting to be successfully transmitted, packets are buffered at the sender. Since buffers are assumed to be of infinite capacity, packets are never lost while travelling through the network.

\section{System Model}

In this section we present the general system model, and we introduce our notation. The general model will be then specialized in Section 5.

\subsection{Sensor Density and Traffic Generation}

Fluid modelling and simulation have proved to be efficient tools for the analysis of complex systems where scalability properties are the key issue. In this paper we use a fluid approach for modelling sensor networks, which is based on the observation that large-scale sensor networks can be represented by a continuous fluid entity distributed on the network area. We identify each point in the plane by means of its coordinates $\boldsymbol{r}=(x, y)$. We denote by $\rho(\boldsymbol{r})$ the sensor density, which is the number of sensors per area unit at point $\boldsymbol{r}$, and can be measured in sensors per square meter. We have that: $\iint \rho(\boldsymbol{r}) \mathrm{d} \boldsymbol{r}=N$. In the case of a disk of unit radius, if we assume that sensors are uniformly distributed and the sink is located at the center of the network area, i.e., $\boldsymbol{S i n} \boldsymbol{k}=(0,0)$, we simply have:

$$
\rho(\boldsymbol{r})=\left\{\begin{array}{cc}
0 & \text { if } \operatorname{dist}(\boldsymbol{r}, \boldsymbol{S i n k})>1 \\
\frac{N}{\pi} & \text { if } \operatorname{dist}(\boldsymbol{r}, \boldsymbol{S i n k}) \leq 1
\end{array}\right.
$$

where $\operatorname{dist}\left(\boldsymbol{r}, \boldsymbol{r}^{\prime}\right)$ denotes the distance between $\boldsymbol{r}$ and $\boldsymbol{r}^{\prime}$.

A sensor in position $\boldsymbol{r}$ generates traffic at rate $\lambda_{s}(\boldsymbol{r})$. By aggregating all traffic generated by sensors over an infinitesimal area centered at point $\boldsymbol{r}$, we can define a generation rate density $\lambda(\boldsymbol{r})$ which depends on the position $\boldsymbol{r}$. This quantity is proportional to both the individual sensor generation rate and the sensor density, $\lambda(\boldsymbol{r})=\lambda_{s}(\boldsymbol{r}) \rho(\boldsymbol{r})$, and can be measured in packet per second per square meter.

\subsection{The Routing Algorithm}

We determine the next hop used by a sensor to send a packet to the sink in a probabilistic way. This accounts for the fact that we know only the node density, not the exact location of the sensors. More specifically, we define as $u\left(\boldsymbol{r}, \boldsymbol{r}^{\prime}\right)$ the probability that a packet generated by a sensor in position $r$ uses as its next hop a sensor in position $\boldsymbol{r}^{\prime}$. Notice that $u\left(\boldsymbol{r}, \boldsymbol{r}^{\prime}\right)$ must be a valid probability density, thus $\iint u\left(\boldsymbol{r}, \boldsymbol{r}^{\prime}\right) \mathrm{d} \boldsymbol{r}^{\prime}=1, \forall \boldsymbol{r}$. In Section 5.1 we will specify $u\left(\boldsymbol{r}, \boldsymbol{r}^{\prime}\right)$ to account for the minimum-energy shortest path algorithm.

\subsection{Total Traffic Rate}

Each sensor can be both a traffic source and a relay for other sensors. We introduce the total traffic rate density $\Lambda(\boldsymbol{r})$ as the sum of the traffic locally generated by the sensors at point $\boldsymbol{r}$, and the traffic relayed for other nodes. The total traffic rate density $\Lambda(\boldsymbol{r})$ can be computed by solving the following integral equation:

$$
\Lambda(\boldsymbol{r})=\lambda(\boldsymbol{r})+\iint \Lambda\left(\boldsymbol{r}^{\prime}\right) u\left(\boldsymbol{r}^{\prime}, \boldsymbol{r}\right) \mathrm{d} \boldsymbol{r}^{\prime}
$$

where $\lambda(\boldsymbol{r})$ accounts for the traffic locally generated, and the integral computes the rate density of the relayed traffic using $u\left(\boldsymbol{r}, \boldsymbol{r}^{\prime}\right)$ introduced above.

\subsection{Success Rate and Power Consumption}

Besides the total traffic rate of sensors at point $\boldsymbol{r}$, two other important metrics have to be defined: the success rate density and the instantaneous power consumption.

Let us denote with $B$ the available channel rate, expressed in packet/s. We define the success rate density $S(\boldsymbol{r})$ as the number of packets that can be successfully transmitted at point $\boldsymbol{r}$ per time unit. This accounts for the fact that the wireless channel is a shared resource, and the fraction of bandwidth enjoyed by a sensor depends on the transmission activity of the other nodes. In particular, we define the interference factor $\hat{I}\left(\boldsymbol{r}, \boldsymbol{r}^{\prime}\right)$ as the fraction of traffic rate at point $\boldsymbol{r}^{\prime}$ that prevents a successful transmission at point $\boldsymbol{r}$. Then, the success rate density at $\boldsymbol{r}$ can be computed as,

$$
S(\boldsymbol{r})=B-\iint \Lambda\left(\boldsymbol{r}^{\prime}\right) \hat{I}\left(\boldsymbol{r}, \boldsymbol{r}^{\prime}\right) \mathrm{d} \boldsymbol{r}^{\prime}
$$

In Section 5.2 we will see how the interference factor can be computed based on the simplifying description of the channel access.

The instantaneous power consumption $P(\boldsymbol{r})$ represents the amount of energy consumed per time unit by the sensors located at point $\boldsymbol{r}$. We denote by $E^{(t x)}\left(\boldsymbol{r}, \boldsymbol{r}^{\prime}\right)$ the energy consumed to transmit a packet from point $\boldsymbol{r}$ to point $\boldsymbol{r}^{\prime}$, by $E^{(r x)}$ the constant energy to receive a packet, and by $P_{i}(\boldsymbol{r})$ the power consumed by a sensor in $r$ while being idle. We can write:

$$
\begin{aligned}
P(\boldsymbol{r}) & =\iint \Lambda(\boldsymbol{r}) E^{(t x)}\left(\boldsymbol{r}, \boldsymbol{r}^{\prime}\right) u\left(\boldsymbol{r}, \boldsymbol{r}^{\prime}\right) \mathrm{d} \boldsymbol{r}^{\prime} \\
& +\iint \Lambda\left(\boldsymbol{r}^{\prime \prime}\right) E^{(r x)} u\left(\boldsymbol{r}^{\prime \prime}, \boldsymbol{r}\right) \mathrm{d} \boldsymbol{r}^{\prime \prime}+P_{i}(\boldsymbol{r})
\end{aligned}
$$

The first term in the r.h.s. of (4) accounts for the power consumption due to packets transmission, the second term takes 
Table 1. Model Parameters

\begin{tabular}{|l|l|}
\hline Parameter & Description \\
\hline$\rho(\boldsymbol{r})$ & Sensor density at $\boldsymbol{r}$ \\
$\lambda(\boldsymbol{r})$ & Traffic generation rate at $\boldsymbol{r}$ \\
$u\left(\boldsymbol{r}, \boldsymbol{r}^{\prime}\right)$ & Probability of sending a packet from $\boldsymbol{r}$ to $\boldsymbol{r}^{\prime}$ \\
$\hat{I}\left(\boldsymbol{r}, \boldsymbol{r}^{\prime}\right)$ & Fraction of traffic generated at $\boldsymbol{r}^{\prime}$ that interferes with $\boldsymbol{r}$ \\
$q(\boldsymbol{r})$ & Expected packet delay at $\boldsymbol{r}$ \\
$E^{(t x)}\left(\boldsymbol{r}, \boldsymbol{r}^{\prime}\right)$ & Energy spent to transmit a packet from $\boldsymbol{r}$ to $\boldsymbol{r}^{\prime}$ \\
$E^{(r x)}$ & Energy spent to receive a packet \\
$P_{i}(\boldsymbol{r})$ & Idle state power consumption at $\boldsymbol{r}$ \\
\hline
\end{tabular}

Table 2. Computed functions

\begin{tabular}{|l|l|c|}
\hline Parameter & Meaning & Eq. \\
\hline$\Lambda(\boldsymbol{r})$ & Total traffic generation rate at $\boldsymbol{r}$ & $(2)$ \\
$S(\boldsymbol{r})$ & Transmission success rate at $\boldsymbol{r}$ & $(3)$ \\
$P(\boldsymbol{r})$ & Instantaneous power consumption at $\boldsymbol{r}$ & $(4)$ \\
$w(\boldsymbol{r})$ & Mean waiting time at $\boldsymbol{r}$ & $(5)$ \\
$h(\boldsymbol{r})$ & Mean number of hops at $\boldsymbol{r}$ & $(6)$ \\
\hline
\end{tabular}

into account the power consumption due to packets reception, and the last term accounts for the power consumption in the idle state.

\subsection{Mean Waiting Time and Mean Number of Hops}

Using expressions similar to (2), we can compute the mean waiting time $w(\boldsymbol{r})$ and the mean number of hops $h(\boldsymbol{r})$ required by a packet originated at point $\boldsymbol{r}$ to reach the sink.

By defining $q(\boldsymbol{r})$ as the average service time (average queueing delay plus transmission time) experienced by a packet at point $\boldsymbol{r}$, we have

$$
\begin{aligned}
w(\boldsymbol{r}) & =q(\boldsymbol{r})+\iint w\left(\boldsymbol{r}^{\prime}\right) u\left(\boldsymbol{r}, \boldsymbol{r}^{\prime}\right) \mathrm{d} \boldsymbol{r}^{\prime} \\
h(\boldsymbol{r}) & =1+\iint h\left(\boldsymbol{r}^{\prime}\right) u\left(\boldsymbol{r}, \boldsymbol{r}^{\prime}\right) \mathrm{d} \boldsymbol{r}^{\prime}
\end{aligned}
$$

Equations (5) and (6) state that the mean waiting time (mean number of hops) at point $\boldsymbol{r}$ can be expressed as the sum of the local service time (one hop) and the mean waiting time (mean number of hops) associated with the next hop. The initial conditions are as follows: $w(0,0)=0$ and $h(0,0)=0$, i.e., in the case of the sink, no queue or next hop are defined. In Section 5.3 we will present an approximate computation of $q(\boldsymbol{r})$ as a function of the success rate and of the total traffic rate at point $\boldsymbol{r}$.

We have summarized the notation introduced to describe the sensor network model in Table 1, while Table 2 reports all variables derived by the solution process.

\section{Model for the considered network scenario}

In this section we explain how the various functions introduced in Section 4 can be specialized to describe the network scenario presented in Section 3. In particular, in Section 5.1 we model the minimum-energy routing scheme; in Section 5.2 we show how the interference factor can be computed based on the Protocol Model; Section 5.3 presents an approximate computation of the average service rate experienced by a packet at point $r$ as a function of the success rate and of the total traffic rate at $\boldsymbol{r}$.

\subsection{The Minimum-Energy Routing Scheme}

Consider the routing scheme defined in Section 3, which selects for each sensor the shortest path minimizing the total amount of energy necessary to deliver a packet to the sink. As a first step, we define the one-hop energy cost $\epsilon\left(\boldsymbol{r}, \boldsymbol{r}^{\prime}\right)$ required to deliver a packet from a source in $r$ to a destination in $\boldsymbol{r}^{\prime}$

$$
\begin{aligned}
\epsilon\left(\boldsymbol{r}, \boldsymbol{r}^{\prime}\right) & =E^{(t x)}\left(\boldsymbol{r}, \boldsymbol{r}^{\prime}\right)+E^{(r x)} \\
& =2\left(E^{(e l e)}+E^{(p r o c)}\right)+C_{d} \cdot \operatorname{dist}\left(\boldsymbol{r}, \boldsymbol{r}^{\prime}\right)^{\eta}
\end{aligned}
$$

where $E^{(e l e)}$ and $E^{(p r o c)}$ account for the consumption due to the transceiver electronics and to processing functions, respectively - these costs are present at both the transmitter and the receiver; $C_{d} \cdot \operatorname{dist}\left(\boldsymbol{r}, \boldsymbol{r}^{\prime}\right)^{\eta}$ is the amplifier cost at the transmitter including the constant factor $C_{d}$, the senderreceiver distance $\operatorname{dist}\left(\boldsymbol{r}, \boldsymbol{r}^{\prime}\right)$, and the exponential decay factor $\eta$ that typically takes values between 2 and 4 .

Next, we specialize the routing probability density $u\left(\boldsymbol{r}, \boldsymbol{r}^{\prime}\right)$, defined in Section 4.2. We denote by $\epsilon_{m}\left(\boldsymbol{r}, \boldsymbol{r}^{\prime}\right)$ the energy required to send a packet from point $r$ to the sink, using a sensor in position $\boldsymbol{r}^{\prime}$ as next hop (multihop energy cost). By using a recursive expression, we can write

$\epsilon_{m}\left(\boldsymbol{r}, \boldsymbol{r}^{\prime}\right)=\left\{\begin{array}{c}\min \left(\epsilon(\boldsymbol{r}, \boldsymbol{S i n k}), \epsilon\left(\boldsymbol{r}, \boldsymbol{r}^{\prime}\right)+\epsilon_{m}\left(\boldsymbol{r}^{\prime}, \boldsymbol{r}^{\prime \prime}\right)\right) \\ \quad \text { if } \operatorname{dist}(\boldsymbol{r}, \boldsymbol{S i n k}) \leq d \\ \epsilon\left(\boldsymbol{r}, \boldsymbol{r}^{\prime}\right)+\epsilon_{m}\left(\boldsymbol{r}^{\prime}, \boldsymbol{r}^{\prime \prime}\right) \\ \text { otherwise }\end{array}\right.$

where $r^{\prime \prime}$ is the point that minimizes the energy required to send a packet from $\boldsymbol{r}^{\prime}$ to the sink. Note that, if point $\boldsymbol{r}$ is within distance $d$ from the sink, either a one-hop or a multihop communication may take place, depending on their energy cost.

When a multihop communication is required, we should determine exactly $\boldsymbol{r}^{\prime \prime}$, which turns out to be exceedingly complicated. Thus, we approximate $\epsilon_{m}\left(\boldsymbol{r}^{\prime}, \boldsymbol{r}^{\prime \prime}\right)$ with $\epsilon_{\text {min }}\left(\boldsymbol{r}^{\prime}\right)$, which is the minimum possible energy needed to send a packet from $\boldsymbol{r}^{\prime}$ to the sink. As described in the Appendix, $\epsilon_{\min }\left(\boldsymbol{r}^{\prime}\right)$ can be computed as,

$$
\begin{aligned}
\epsilon_{\min }\left(\boldsymbol{r}^{\prime}\right)= & 2 n\left(E^{(\text {ele })}+E^{(\text {proc })}\right)+C_{d} \frac{\operatorname{dist}\left(\boldsymbol{r}^{\prime}, \boldsymbol{S i n k}\right)^{\eta}}{n^{\eta-1}} \\
& \text { with } n=\max \left(n^{*},\left\lceil\frac{\operatorname{dist}\left(\boldsymbol{r}^{\prime}, \boldsymbol{S i n} \boldsymbol{k}\right)}{d}\right\rceil\right)
\end{aligned}
$$


where $n^{*}$ is the integer number of hops such that $2 n^{*}\left(E^{(e l e)}+E^{(\text {proc })}\right)+C_{d} \frac{\operatorname{dist}\left(\boldsymbol{r}^{\prime}, \boldsymbol{S i n k}\right)^{\eta}}{\left(n^{*}\right)^{\eta-1}}$ is minimum.

Given the sensor density $\rho(\boldsymbol{r})$, we compute the probability density $p_{s}(\boldsymbol{r})$ of finding a sensor at point $\boldsymbol{r}$ as,

$$
p_{s}(\boldsymbol{r})=\frac{\rho(\boldsymbol{r})}{\iint \rho\left(\boldsymbol{r}^{\prime}\right) \mathrm{d} \boldsymbol{r}^{\prime}}
$$

i.e., the probability to find a sensor in position $\boldsymbol{r}$ is directly proportional to the sensor density, and it is normalized so that its integral over the entire area sums to 1 . Then we compute the probability $p_{E}(\boldsymbol{r}, e)$ that the energy required to send a packet from point $\boldsymbol{r}$ to the sink is equal to $e$, and the corresponding cumulative distribution $F_{E}(\boldsymbol{r}, e)$ :

$$
\begin{aligned}
& p_{E}(\boldsymbol{r}, e)=\oint_{\mathbf{r}^{\prime} ; \epsilon_{m}\left(\mathbf{r}, \mathbf{r}^{\prime}\right)=e} p_{s}\left(\boldsymbol{r}^{\prime}\right) \mathrm{d} \boldsymbol{r}^{\prime} \\
& F_{E}(\boldsymbol{r}, e)=\int_{0}^{e} p_{E}\left(\boldsymbol{r}, e^{\prime}\right) \mathrm{d} e^{\prime}
\end{aligned}
$$

Equation (11) states that the probability of having a minimum energy path requiring expenditure $e$ corresponds to the sum of the probabilities of finding a sensor at all points through which a packet can be transferred to the sink with energy cost $e$. Since there may be many points $\boldsymbol{r}^{\prime}$ around $\boldsymbol{r}$ that, if used as relays, would imply an energy consumption $e$ to send a packet to the sink, $p_{E}(\boldsymbol{r}, e)$ is expressed as an integral over an iso-energy line.

Now, consider a source node in position $r$ and the fact that there are $N-1$ sensors that could be used as relays by the source (i.e., all sensors except the source itself). The energy required to send a packet to the sink using one of them as next hop is distributed according to (11). Thus, the probability density $P_{m E}(\boldsymbol{r}, e)$ that the minimum energy required to send a packet to the sink is equal to $e$ can be computed as,

$$
\begin{aligned}
F_{m E}(\boldsymbol{r}, e) & =1-\left[1-F_{E}(\boldsymbol{r}, e)\right]^{N-1} \\
P_{m E}(\boldsymbol{r}, e) & =\frac{\mathrm{d} F_{m E}(\boldsymbol{r}, e)}{\mathrm{d} e}
\end{aligned}
$$

Then we define $p_{s ; \mathbf{r}}\left(\boldsymbol{r}^{\prime} \mid e\right)$ as the probability of finding a sensor in position $\boldsymbol{r}^{\prime}$ that can be used as next hop by a sensor in $\boldsymbol{r}$ to send a packet to the sink with energy expenditure $e$, conditioned to the value of the minimum required energy. $p_{s ; \mathbf{r}}\left(\boldsymbol{r}^{\prime} \mid e\right)$ is given by,

$$
p_{s ; \mathbf{r}}\left(\boldsymbol{r}^{\prime} \mid e\right)=\left\{\begin{array}{cl}
\frac{p_{s}\left(\boldsymbol{r}^{\prime}\right)}{p_{E}(\boldsymbol{r}, e)} & \text { if } \epsilon_{m}\left(\boldsymbol{r}, \boldsymbol{r}^{\prime}\right)=e \\
0 & \text { otherwise }
\end{array}\right.
$$

i.e., $p_{s ; \mathbf{r}}\left(\boldsymbol{r}^{\prime} \mid e\right)$ is equal to 0 if the energy required to send a packet from position $\boldsymbol{r}$ to the sink, using as next hop a relay in position $\boldsymbol{r}^{\prime}$, is different from $e$; otherwise it is equal to the probability of finding a sensor in $\boldsymbol{r}^{\prime}$, normalized by the total probability of finding a sensor that requires energy $e$.

Finally, using (14) and (15), the routing function $u\left(\boldsymbol{r}, \boldsymbol{r}^{\prime}\right)$ is given by,

$$
u\left(\boldsymbol{r}, \boldsymbol{r}^{\prime}\right)=p_{s ; \mathbf{r}}\left(\boldsymbol{r}^{\prime} \mid e\right) P_{m E}(\boldsymbol{r}, e)
$$

\subsection{A Simple Channel Access Model}

The Protocol Model introduced in Section 3 provides the necessary condition for a packet to be successfully transmitted to another node within distance $d$ from the sender. Using our fluid representation, we say that the transmission from a sensor in position $\boldsymbol{r}^{\prime}$ interferes with a transmission from a sensor in $\boldsymbol{r}$, destined to another node in position $\boldsymbol{r}^{\prime \prime}$, if and only if $\boldsymbol{r}^{\prime \prime}$ is within distance $d$ from $\boldsymbol{r}^{\prime}$ (i.e., $\left.\operatorname{dist}\left(\boldsymbol{r}^{\prime}, \boldsymbol{r}^{\prime \prime}\right) \leq d\right)$. Thus, the interference factor $\hat{I}\left(\boldsymbol{r}, \boldsymbol{r}^{\prime}\right)$ introduced in Section 4.4 is given by,

$$
\hat{I}\left(\boldsymbol{r}, \boldsymbol{r}^{\prime}\right)=\iint_{\mathbf{r}^{\prime \prime}: \operatorname{dist}\left(\mathbf{r}^{\prime}, \mathbf{r}^{\prime}\right) \leq d} u\left(\boldsymbol{r}, \boldsymbol{r}^{\prime \prime}\right) \mathrm{d} \boldsymbol{r}^{\prime \prime}
$$

\subsection{The Total Service Time}

The total service time introduced in Section 4.5 can be approximated by using a simple birth/death model. Let us define $\alpha=\frac{\Lambda(\boldsymbol{r})}{B \cdot \rho(\boldsymbol{r})}$ and $\beta=\frac{1}{B}\left(B-S(\boldsymbol{r})-\frac{\Lambda(\boldsymbol{r})}{\rho(\boldsymbol{r})}\right)$. The variable $\alpha$ represents the probability of having a packet to transmit at a node in position $r$, because it has been either locally generated or received from another sensor. The variable $\beta$ represents the probability of not being able to successfully transmit a packet, due to other nodes transmitting within distance $d$ from the intended receiver. Note that, since the expression of the success rate density also includes the traffic locally generated at point $\boldsymbol{r}$ (see (3)), this has to be subtracted.

We approximate the number of packets stored in the buffer of a sensor in position $\boldsymbol{r}$ by a birth/death process: the birth rate is equal to $b=\alpha \beta$, and the death rate is equal to $u=(1-\alpha)(1-\beta)$. Indeed, the number of queued packets grows if a new packet is either generated or received $(\alpha)$, but cannot be successfully sent over the wireless channel $(\beta)$; it is decremented by one if no packet is generated or received $(1-\alpha)$, and the transmission is successful $(1-\beta)$ so that a packet can be forwarded. Note that this is an approximation, as in reality the number of packets may increase by two units in a single step if both a new packet is generated and a packet is received from another sensor; however our approximation works well for most of the cases. Using this 
approach we can easily compute the mean queue length as,

$\bar{n}(\boldsymbol{r})=\frac{b}{u-b}=\frac{\alpha \beta}{1-\alpha-\beta}=\frac{1}{B} \frac{\frac{\Lambda(\boldsymbol{r})}{\rho(\boldsymbol{r})}\left(B-S(\boldsymbol{r})-\frac{\Lambda(\boldsymbol{r})}{\rho(\boldsymbol{r})}\right)}{S(\boldsymbol{r})}$

Finally, applying Little's formula, the expected queueing delay $q(\boldsymbol{r})$ is given by,

$$
q(\boldsymbol{r})=\frac{1}{B}+\frac{\bar{n}(\boldsymbol{r})}{\frac{\Lambda(\boldsymbol{r})}{\rho(\boldsymbol{r})}}=\frac{1}{B}\left(1+\frac{B-S(\boldsymbol{r})-\frac{\Lambda(\boldsymbol{r})}{\rho(\boldsymbol{r})}}{S(\boldsymbol{r})}\right)
$$

\section{Performance Evaluation}

Here, we first validate our model via simulation in the case where $N=500$ and the sensors are uniformly distributed over a disk of unit radius, with the sink being located at the center of the disk. Then, we increase the network size by setting $N$ to 1000 , and derive several performance metrics of interest.

\subsection{Model Validation}

The results derived from the fluid model are compared against detailed simulations of the same system. We have used an ad-hoc, discrete-time simulator, based on the same assumptions as described in Section 3 and where time is discretized into time slots of unit duration.

At the beginning of the simulation we generate a random (connected) topology, and we apply Dijkstra algorithm to compute once and for all the shortest path route available to each node according to the energy cost defined in Section 3. To simulate the ideal MAC protocol described in Section 5.2, we have adopted the following technique. At the beginning of each time slot, all sensors can potentially transmit or receive a packet during the slot. To solve the contention on the channel, we extract a random permutation of the indexes $1,2, \ldots, N$ associated to the sensor nodes. Then, we inspect each sensor in the order resulting from the permutation. If the sensor is able to transmit a packet to its next hop, it is allowed to do so. Furthermore, all nodes within distance $d$ from the transmitting sensor are no more allowed to receive until the beginning of the next slot. Similarly, all nodes within distance $d$ from the receiving next hop are not allowed to transmit during the current slot. This way, we randomly and fairly choose the transmissions that take place in the network during each time slot.

Since the simulation results are obtained for a particular instance of sensors deployment, the correct validation methodology requires averaging the simulation predictions over a large number of deployment realizations. We chose to run 100 simulation experiments for each set of system parameters, and to average the outcome of each experiment.

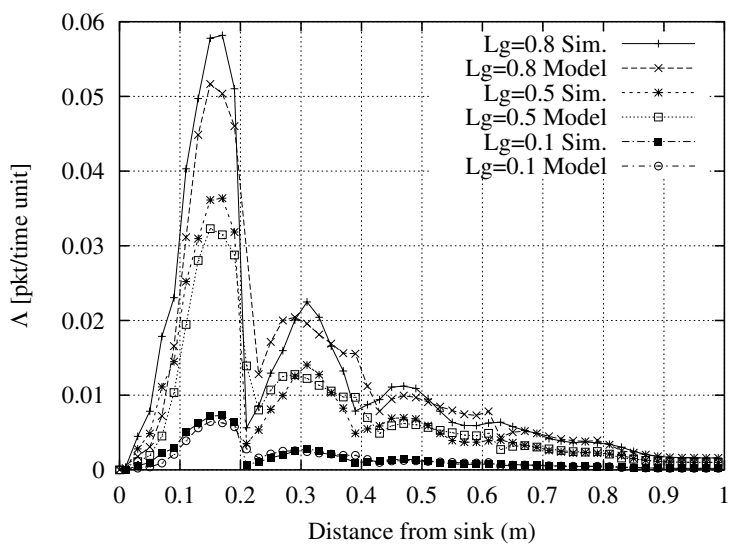

Figure 1. Average total traffic rate per sensor, $\Lambda$, versus the node distance from the sink, for different values of $L_{g}$. Analytical and simulation results are compared

Each simulation experiment discards an initial transient period that we set equal to 5,000 time slots. The length of each run is equal to 50,000 time slots. Furthermore, while our model allows for the computation of the spatial distribution of performance metrics, to ease the interpretation of results as well as the comparison against simulation outcomes, we averaged the performance metrics over the space points at the same distance from the sink. Doing so we obtained 2D graphs of the performance metrics as functions of the distance from the sink.

We set the system parameters as follows: $d=0.2$, $N=500, B=1, C_{d}=0.057 \mathrm{~mJ}, E^{(e l e)}=E^{(\text {proc })}=0.24 \mathrm{~mJ}$, and $P_{i}=18 \mathrm{~mW}$. Let us denote by $L_{g}$ the integral over the network area of the traffic generation rates $\lambda_{s}(\boldsymbol{r})$ of the sensors. To validate our model under a wide range of system loads, we consider three values of $L_{g}: 0.1,0.5$, and $0.8 .^{2}$ We assume that the traffic generation rate is uniform across the network, thus $\lambda_{s}(\boldsymbol{r})=L_{g} / N, \forall \boldsymbol{r}$.

First of all, we investigate the total traffic rate per sensor, $\Lambda(\boldsymbol{r})$, which accounts for both the packets generated by the node and the relay traffic. Figure 1 reports the behavior of $\Lambda(\boldsymbol{r})$ versus the node distance from the sink. We observe that the traffic rate (and, hence, energy consumption) is not evenly distributed across the network; indeed, nodes closer to the sink have to relay a larger amount of traffic than peripheral sensors. This is the well known problem of data implosion at the sink [17] that affects multipoint-to-point communications and results into unfairness among the network nodes. We also observe that traffic rate per sensor di-

\footnotetext{
${ }^{2}$ Note that, since the maximum theoretical value of network capacity cannot exceed 1 (the sink cannot receive more than one packet per time slot), the total network load $L_{g}$ is limited to the interval $(0,1]$
} 


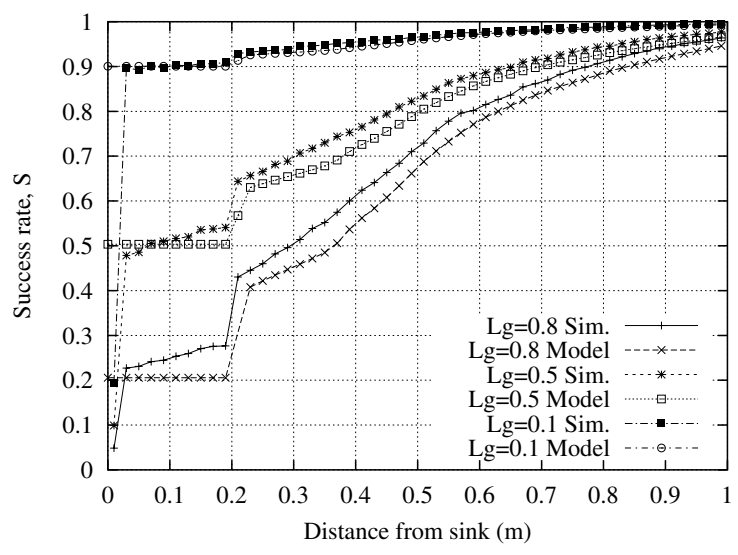

Figure 2. Success rate per sensor as a function of the node distance from the sink, for different values of $L_{g}$. Analytical and simulation results are compared

minishes significantly at distances close to multiples of the maximum radio range $d$. This is due to the routing strategy adopted, which selects the path minimizing the overall energy cost to send a packet to the sink. Notice that, with our choice of values for $C_{d}, E^{(e l e)}$ and $E^{(p r o c)}$, it turns out that the fixed cost required to transmit a packet over one hop is much larger than the variable cost due to the amplifier, even if sending at the maximum possible distance $d$. As a consequence, routes are primarily selected on the basis of the minimum hop count. Among all routes having the minimum number of hops, the one minimizing the variable cost due to the amplifier is preferred. Finally, looking at Figure 1 , we observe that the model is able to accurately predict also the particular shape of the curves obtained for different values of $L_{g}$.

The success rate per sensor, $S(\boldsymbol{r})$, is plotted in Figure 2 as a function of the node distance from the sink. The success rate grows as the distance from the sink increases; indeed, sensors in the radio range of the sink are those experiencing the highest interference factor, which approaches the value of $L_{g}$. The model is again very accurate in predicting the system performance at all considered loads.

Figure 3 shows the data latency versus the source distance from the sink. Data latency is the delay elapsed from the time at which a packet is generated by some network node to the time at which the packet is delivered to the sink. We observe that the latency increases with the distance from the sink as well as with the value of $L_{g}$, as expected. The data latency is very well approximated by our birth-death process whose parameters are derived from the traffic rate and success rate density. The matching is excellent for all considered values of $L_{g}$.

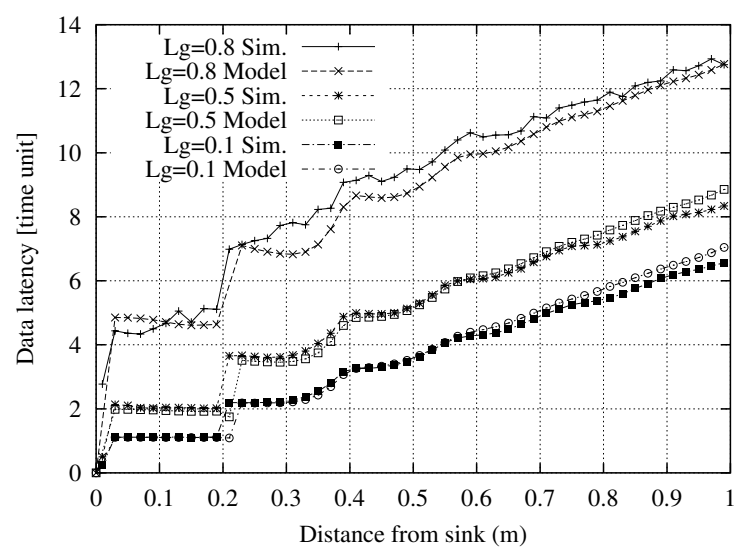

Figure 3. Average data latency vs. the node distance from the sink, for different values of $L_{g}$. Analytical and simulation results are compared

We have validated our model also for larger values of $N$ (up to the limit of the simulator) and for different values of $d$ (ranging from 0.1 to 0.4 ). The matching between the simulation results and the model predictions is excellent for all of the combinations of system parameters.

\subsection{Model Exploitation}

In this section, we present an example of how our model can be used to investigate different strategies in sensor network design and planning. Many other issues relevant to sensor networks could be studied, and different architectural solutions and parameter settings could be explored (see Section 7 for a discussion on other possible applications of our model).

In the previous section, we observed the effect of data implosion at the sink, and the consequent unfairness among the sensors. Such an unfairness may lead to an uneven battery discharge across the network and to a severe penalty in the network capacity, resulting in degraded performance also at low loads (few congested nodes can introduce high queueing delays adversely impacting the average data transfer latency).

Several solutions to this problem are possible; here we explore the possibility to act on the network deployment so as to mitigate the data implosion phenomenon. We consider that sensors can be deployed over the network area in four different ways, labelled, respectively, as 'Homogeneous', ' $C_{2}$ ', ' $C_{3}$ ', and ' $C_{4}$ '. The case 'Homogeneous' corresponds to a uniform deployment of the nodes over the disk of unit radius. ' $C_{x}$ ' $(x=2,3,4)$ represents the case where the sensor density within a ring from distance 0.1 to distance 
0.3 from the sink is increased by a factor $x$ w.r.t. the uniform deployment, while the nodes outside the ring are deployed as in the 'Homogeneous' case.

Unless differently specified, we assume that the total traffic generation rate of the network, $L_{g}$, is fixed to 0.5 , the remaining parameters are set as in Section 6.1. In the 'Homogeneous' network we set the number of nodes, $N$, and the sensor generation rate, $\lambda_{s}$ to 1000 and 0.0005 , respectively, so that we have $L_{g}=0.5$. Under the ' $C_{x}$ ' deployment, the nodes outside the ring have a generation rate of 0.0005 , while the generation rate of the sensors within the ring is $x(x=2,3,4)$ times lower; $N$ is properly varied so as to maintain $L_{g}=0.5$. This solution is motivated by the fact that the production cost of a sensor is typically modest; therefore, increasing the number of nodes may be a good solution to avoid service inefficiencies or network disconnections due to some nodes that have prematurely exhausted their battery.

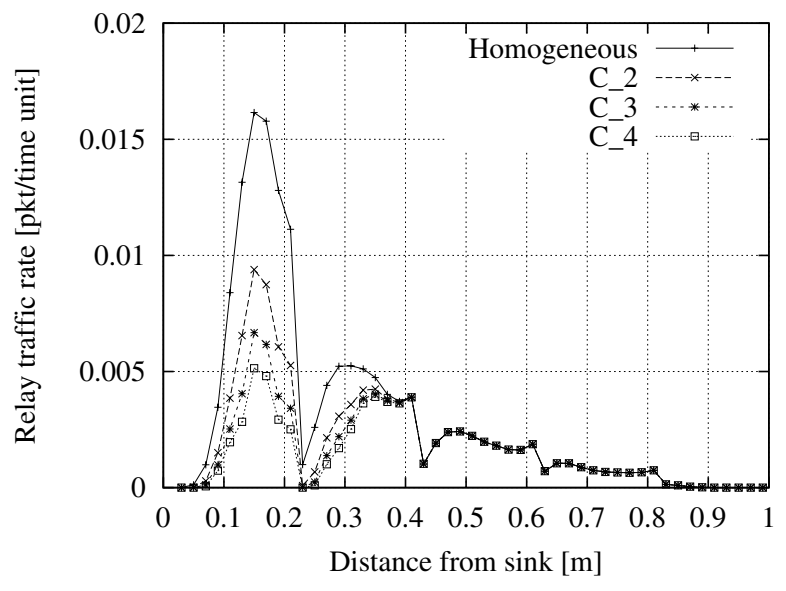

Figure 4. Average relay traffic rate per sensor versus the node distance from the sink, under different node deployments

Figures 4 and 5 show, respectively, the relay traffic rate and the energy consumption rate per node, as functions of the sensor distance from the sink. Figure 4 highlights how the load due to relayed traffic becomes more evenly distributed across the network as we pass from the 'Homogeneous' to the ' $C_{4}$ ' configuration. This implies that increasing the node density within an appropriate area around the sink significantly helps in balancing the traffic rate among sensors, thus improving fairness. Finally, Figure 5 presents the improvement in the distribution of energy consumption under the $C_{x}$ deployments. Indeed, by making the traffic rate more balanced, the $C_{x}(x=2,3,4)$ configurations enable us to even the energy depletion of sensors as $x$ increases. This is also confirmed by the results reported in

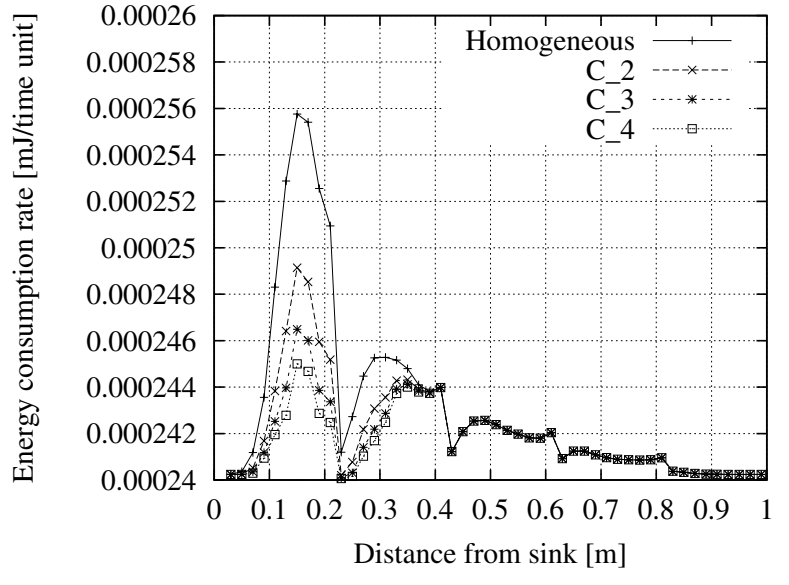

Figure 5. Average energy consumption rate per sensor as a function of the node distance from the sink, under different network deployments

Table 3. Coefficient of variation of the energy consumption rate and network lifetime under different sensor deployments

\begin{tabular}{|c|c|c|c|}
\hline Configuration & $\begin{array}{c}\text { CV of } \\
\text { the energy } \\
\text { consumption rate }\end{array}$ & $\begin{array}{c}\text { Lifetime [h] } \\
\text { (first node dies) }\end{array}$ & $\begin{array}{c}\text { Lifetime [h] } \\
\text { (disconnection) }\end{array}$ \\
\hline \hline Homogeneous & 0.010786 & 1019.08 & 1139.33 \\
\hline$C=2$ & 0.006461 & 1066.19 & 1140.71 \\
\hline$C=3$ & 0.005262 & 1086.43 & 1140.69 \\
\hline$C=4$ & 0.004786 & 1097.85 & 1140.67 \\
\hline
\end{tabular}

Table 3, presenting the coefficient of variation of the energy consumption rate and the network lifetime, expressed in hours.

Results on energy consumption have been derived assuming that the initial energy availability at each sensor is equal to $1000 \mathrm{~J}$, corresponding to a battery duration of about 1080 hours. The results are presented for two different definitions of network lifetime. In one case we consider the time that elapses from the instant when the network starts functioning till the first node runs out of energy; in the other case, we take as ending time the instant when all sensors within a ring of width $d$ (at any distance from the sink) exhaust their energy. Note that the latter definition provides a loose upper bound on the time instant at which the network gets disconnected, i.e., one or more nodes no longer can send a packet to the sink. In fact, in the case of a symmetric network scenario like the one considered here, nodes outside the ring can no longer send a packet to any node in the area comprising the sink.

As a last comment, we would like to mention that results equivalent to the ones shown above have been obtained when the number of sensors and the traffic generation rate 
of each sensor are constants, and we increase the node density within the ring area by a factor $x(x=2,3,4)$ (doing so, we vary the total traffic generation rate $L_{g}$ ).

\subsection{Model Solution Complexity}

The computational cost for the derivation of numerical results is extremely small and independent of the number of sensors. The CPU time needed for the computation of the model predictions depends on the solution algorithm we implemented. We discretized the disk of unit radius using $N_{p}=2500$ points, thus obtaining a radial discretization step equal to $\frac{1}{\sqrt{N_{p}}}$. Increasing the number of discretization points resulted in a slightly increased accuracy of the model predictions at the cost of an increased CPU time required for the model solution.

The CPU time required to obtain the model predictions for any set of system parameters we presented in this paper was only a few seconds (usually ten), while the simulation experiments took much longer to complete. Indeed, the simulation time grows more than linearly with the number of nodes, allowing to consider at most a few hundreds of nodes.

\section{Discussion and Future Work}

Our spatial, fluid approach provides a very powerful and flexible framework that can be used to study several important issues in wireless sensor networks. We present below some aspects that can be subject of future research.

(i) One of the most effective ways to save energy in sensor networks is the use of sleep modes, by which nodes enter low-power operational states. We can easily extend the model so as to represent the case where sensors have an active/sleep behavior, by means of a probabilistic approach.

(ii) A transient analysis of the system can be performed, which would allow us to track the sensors' battery discharge as time goes on, and to consider the behavior of the nodes dependent on their residual energy. A transient analysis would also enable us to precisely derive the time instant at which the network gets disconnected, and evaluate the network performance as more and more sensors exhaust their battery.

(iii) The model can be easily extended to consider the presence of multiple sinks and the possibility of performing data aggregation at the sensor nodes.

(iv) Various routing strategies, besides the one considered in this paper, could be applied; for example, geographic or QoS-based routing schemes, both with and without data aggregation.

\section{Conclusions}

In this paper, we presented a new methodology to analyze the behavior of large-scale sensor networks. Our approach is based on a fluid representation of all quantities that depend on the specific location within the network topology, and by probabilistic functions to characterize the individual nodes behavior. The model predictions have been validated against results obtained through an ad-hoc simulator under several system loads; the model proved to be extremely accurate for the computation of all considered performance metrics. Furthermore, the model solution complexity does not depend on the network size but on the discretization technique only, thus allowing for the investigation of large-scale sensor networks at very low computational cost.

\section{References}

[1] X. Zeng, R. Bagrodia, and M. Gerla, "Glomosim: A Library for Parallel Simulation of Large-scale Wireless Networks," Workshop on Parallel and Distributed Simulation, 1998.

[2] G. Simon, P. Volgyesi, M. Maroti, and A. Ledeczi, "Simulation-based Optimization of Communication Protocols for Large-scale Wireless Sensor Networks," 2003, http://www.isis.vanderbilt.edu/projects/ nest/prowler/

[3] P. Levis, N. Lee, M. Welsh, and D. Culler, "Tossim: Accurate and Scalable Simulation of Entire Tinyos Applications," First ACM Conference on Embedded Networked Sensor Systems, Berkeley, 2003.

[4] O. Dousse, P. Mannersalo, and P. Thiran, "Latency of Wireless Sensor Networks with Uncoordinated Power Saving Mechanisms," ACM MobiHoc, Tokio, Japan, June 2004.

[5] C. Florens and R. McEliece, "Packet Distribution Algorithms for Sensor Networks," IEEE INFOCOM, San Francisco, CA, Mar. 2003.

[6] B. Krishnamachari, D. Estrin, and S. Wicker, "Modelling Data-Centric Routing in Wireless Sensor Networks," IEEE INFOCOM, New York, NY, June 2002.

[7] M. Zorzi and R. R. Rao, "Geographic Random Forwarding (GeRaF) for Ad Hoc and Sensor Networks: Energy and Latency Performance," IEEE Trans. on Mobile Computing, vol. 2, pp. 337-347, Oct.-Dec. 2003

[8] T. Melodia, D. Pompili, I. F. Akyildiz, ”Optimal Local Topology Knowledge for Energy Efficient Geographical Routing in Sensor Networks," IEEE INFOCOM, Hong Kong, Mar. 2004.

[9] R. Cristescu, B. Beferull-Lozano, M. Vetterli, "On Network Correlated Data Gathering," IEEE INFOCOM, Hong Kong, China, Mar. 2004.

[10] S. Shakkottai, R. Srikant, and N. B. Shroff, "Unreliable Sensor Grids: Coverage, Connectivity and Diameter," IEEE INFOCOM, San Francisco, CA, Apr. 2003. 
[11] B. Liu and D. Towsley, "A Study on the Coverage of Largescale Sensor Networks," 1st IEEE International Conference on Mobile Ad-hoc and Sensor Systems (MASS 2004), Fort Lauderdale, FL, Oct. 2004.

[12] O. Dousse, F. Baccelli, P. Thiran, "Impact of Interference on Connectivity in Ad Hoc Networks," IEEE INFOCOM, San Francisco, CA, Apr. 2003.

[13] P. K. Gopala and H. El Gamal, "On the Scaling Laws of Multi-modal Wireless Sensor Networks," IEEE INFOCOM, Hong Kong, Mar. 2004

[14] C.-F. Chiasserini and M. Garetto, "Modeling the Performance of Wireless Sensor Networks," IEEE INFOCOM, Hong Kong, Mar. 2004.

[15] W. Rabiner Heinzelman, A. Chandrakasan, and H. Balakrishnan, "Energy-Efficient Communication Protocol for Wireless Microsensor Networks," 33rd International Conference on System Sciences (HICSS ’00), Jan. 2000.

[16] P. Gupta and P.R. Kumar, "The Capacity of Wireless Networks," IEEE Trans. on Information Theory, vol.46, Mar. 2000.

[17] J. Kulik, W. Rabiner Heinzelman, and H. Balakrishnan, "Negotiation-based Protocols for Disseminating Information in Wireless Sensor Networks, ACM/IEEE MobiCom '99, Seattle, WA, Aug. 1999.

\section{Appendix}

Here we prove that the minimum possible energy required to send a packet to the sink from a point $\boldsymbol{r}$ is given by (9). We carry on the proof into three steps: first we prove that all sensors on the route must be aligned; next we prove that the distance between two consecutive sensors must be the same; finally we show how to determine the minimum number of hops required to reach the sink.

As a first step, assume that the minimum number of hops required to reach the sink from point $r$ is known and equal to $n$. Let $\sigma(\boldsymbol{r})=\left(\boldsymbol{r}_{0}, \boldsymbol{r}_{1}, \ldots, \boldsymbol{r}_{k}, \ldots \boldsymbol{r}_{n}\right)$ (with $\boldsymbol{r}_{0}=\boldsymbol{r}$ and $\boldsymbol{r}_{n}=\boldsymbol{S i n \boldsymbol { k } )}$ be a sequence of points, such that $\operatorname{dist}\left(\boldsymbol{r}_{j}, \boldsymbol{r}_{j+1}\right) \leq d, 0 \leq j<n$. The energy required to send a packet from $\boldsymbol{r}$ to the sink using as relays the sensors located at the points of $\sigma(\boldsymbol{r})$ is given by,

$$
\begin{aligned}
E(\sigma(\boldsymbol{r})) & =\sum_{j=0}^{n-1}\left[2\left(E^{(e l e)}+E^{(\text {proc })}\right)+C_{d} \cdot \operatorname{dist}\left(\boldsymbol{r}_{j}, \boldsymbol{r}_{j+1}\right)^{\eta}\right] \\
& =2 n\left(E^{(e l e)}+E^{(p r o c)}\right)+C_{d} \sum_{j=0}^{n-1} \operatorname{dist}\left(\boldsymbol{r}_{j}, \boldsymbol{r}_{j+1}\right)^{\eta}
\end{aligned}
$$

We can prove that on the minimum energy path all points in $\sigma(\boldsymbol{r})$ stay on the line connecting $\boldsymbol{r}$ to the sink. Suppose for simplicity that all points are aligned except point $\boldsymbol{r}_{k}$. Let us call $\boldsymbol{r}_{k}^{\prime}$ the projection of point $\boldsymbol{r}_{k}$ on the line. Notice that $\boldsymbol{r}^{\prime}{ }_{k}$ is aligned with the other points. Let us call $\sigma^{\prime}(\boldsymbol{r})=\left(\boldsymbol{r}_{0}, \ldots, \boldsymbol{r}_{k}^{\prime}, \ldots, \boldsymbol{r}_{n}\right)$. By construction, we have that: $\operatorname{dist}\left(\boldsymbol{r}_{k-1}, \boldsymbol{r}_{k}^{\prime}\right)^{\eta} \leq \operatorname{dist}\left(\boldsymbol{r}_{k-1}, \boldsymbol{r}_{k}\right)^{\eta}$ and $\operatorname{dist}\left(\boldsymbol{r}_{k}^{\prime}, \boldsymbol{r}_{k+1}\right)^{\eta} \leq \operatorname{dist}\left(\boldsymbol{r}_{k}, \boldsymbol{r}_{k+1}\right)^{\eta}$. We define the quantity

$A=2 n\left(E^{(e l e)}+E^{(\text {proc })}\right)+C_{d} \sum_{j=0, j \neq k-1, k}^{n-1} \operatorname{dist}\left(\boldsymbol{r}_{j}, \boldsymbol{r}_{j+1}\right)^{\eta}$

Then, we can show that $E\left(\sigma^{\prime}(\boldsymbol{r})\right) \leq E(\sigma(\boldsymbol{r}))$,

$E\left(\sigma^{\prime}(\boldsymbol{r})\right)=A+C_{d}\left[\operatorname{dist}\left(\boldsymbol{r}_{k-1}, \boldsymbol{r}^{\prime}{ }_{k}\right)^{\eta}+\operatorname{dist}\left(\boldsymbol{r}_{k}^{\prime}, \boldsymbol{r}_{k+1}\right)^{\eta}\right] \leq$ $A+C_{d}\left[\operatorname{dist}\left(\boldsymbol{r}_{k-1}, \boldsymbol{r}_{k}\right)^{\eta}+\operatorname{dist}\left(\boldsymbol{r}_{k}, \boldsymbol{r}_{k+1}\right)^{\eta}\right]=E(\sigma(\boldsymbol{r}))$.

Now we can assume that all points of the route are aligned and prove that the distance between two consecutive points is constant and equal to $\alpha=\frac{\operatorname{dist}\left(\boldsymbol{r}_{0}, \boldsymbol{r}_{n}\right)}{n}$. Consider a generic sequence $\sigma(\boldsymbol{r})$ of $n$ points aligned on the same segment. We indicate the distance between two consecutive points as: $\operatorname{dist}\left(\boldsymbol{r}_{j}, \boldsymbol{r}_{j+1}\right)=\alpha_{j}$, with $0 \leq j \leq n-2$, and the one between the last two points as: $\operatorname{dist}\left(\boldsymbol{r}_{n-1}, \boldsymbol{r}_{n}\right)=$ $\operatorname{dist}\left(\boldsymbol{r}_{0}, \boldsymbol{r}_{n}\right)-\sum_{j=0}^{n-2} \alpha_{j}$. Then, we have
$E(\sigma(r))=2 n\left(E^{e l e}+E^{\text {proc }}\right)+$
$+C_{d}\left[\sum_{j=0}^{n-2} \alpha_{j}^{\eta}+\left(\operatorname{dist}\left(r_{0}, r_{n}\right)-\sum_{j=0}^{n-2} \alpha_{j}\right)^{\eta}\right]$

The partial derivatives of $E(\sigma(r))$ along $\alpha_{j}, 0 \leq j \leq n-2$, are as follows,

$\frac{\partial E(\sigma(r))}{\partial \alpha_{j}}=C_{d}\left[\eta \alpha_{j}^{\eta-1}-\eta\left(\operatorname{dist}\left(r_{0}, r_{n}\right)-\sum_{j=0}^{n-2} \alpha_{j}\right)^{\eta-1}\right]$

It can be easily seen that all partial derivatives are equal to 0 if the distance between any two consecutive nodes $\alpha_{j}$ is constant and equal to $\alpha=\frac{\operatorname{dist}\left(\boldsymbol{r}_{0}, \boldsymbol{r}_{n}\right)}{n}$.

So far we have proven that along the minimum energy path, all points are aligned and equidistant. If the number of points is equal to $n$, then we have

$E_{\text {min }}(\boldsymbol{r}, n)=2 n\left(E^{(e l e)}+E^{(\text {proc })}\right)+C_{d} \frac{\operatorname{dist}(\boldsymbol{r}, \boldsymbol{S i n} \boldsymbol{k})^{\eta}}{n^{\eta-1}}$

Now we have to determine a valid value for $n$ that minimizes the energy cost of the path. If we consider (20) to be continuous on $n$, we can compute its derivative and obtain the minimum of the function for $n=$ $\operatorname{dist}\left(\boldsymbol{r}_{0}, \boldsymbol{r}_{n}\right) \sqrt[\eta]{\frac{C_{d}}{2\left(E^{(e l e)}+E^{(\text {proc })}\right)}}$. Since $n$ must be an integer, we define $n^{*}$ to be the nearest integer to $n$ that minimizes the energy. However, we must also satisfy the constraint that $\alpha=\frac{\operatorname{dist}(\boldsymbol{r}, \text { Sink })}{n} \leq d$, that is $n \geq\left\lceil\frac{\operatorname{dist}(\boldsymbol{r}, \text { Sink })}{d}\right\rceil$. 